\title{
Cannabinoid WIN55, 212-2 induces cell cycle arrest and inhibits the proliferation and migration of human BEL7402 hepatocellular carcinoma cells
}

\author{
DACAI XU ${ }^{1}$, JIANGLIN WANG ${ }^{1}$, ZHENKANG ZHOU ${ }^{2}$, ZHIWEI HE $^{3}$ and QING ZHAO ${ }^{1}$ \\ ${ }^{1}$ Department of Biochemistry, Guangzhou Medical University, Guangzhou, Guangdong 510182; \\ ${ }^{2}$ School of Mathematics, South China University of Technology, Guangzhou, Guangdong 510640; \\ ${ }^{3}$ Guangdong Provincial Key Laboratory of Medical Molecular Diagnostics, Dongguan, Guangdong 523376, P.R. China
}

Received December 17, 2014; Accepted September 22, 2015

DOI: $10.3892 / \mathrm{mmr} .2015 .4477$

\begin{abstract}
Hepatocellular carcinoma (HCC) is the leading cause of cancer-associated mortality worldwide; however, only limited therapeutic treatments are currently available. The present study aimed to investigate the effects of cannabinoids as novel therapeutic targets in HCC. In addition, the mechanism underlying the effects of a synthetic cannabinoid, WIN55, 212-2, on the BEL7402 HCC cell line was investigated. The results demonstrated that WIN55, 212-2 induced cell cycle arrest of the BEL7402 cells at the G0/G1 phase via cannabinoid receptor 2 (CB2)-mediated downregulation of phosphorylated-extracellular signal-regulated kinases (ERK)1/2, upregulation of p27, and downregulation of cyclin D1 and cyclin-dependent kinase 4. Furthermore, inhibition of CB2 with the CB2 antagonist AM630 abrogated WIN55, 212-2-induced cell cycle arrest. Inhibition of ERK1/2 also resulted in cell cycle dysregulation and cell cycle arrest at the G0/G1 phase, which subsequently resulted in cell growth inhibition. In addition, the present study detected a significant reduction in matrix metalloproteinase-9, retinoblastoma protein and E2F1 expression, and migration inhibition by WIN treatment. These results suggested that cannabinoid receptor agonists, including WIN, may be considered as novel therapeutics for the treatment of HCC.
\end{abstract}

\section{Introduction}

Hepatocellular carcinoma (HCC) is one of the most common types of malignancy in China $(1,2)$. HCC presents a serious threat to human health due to its rising incidence, high metastatic recurrence and mortality rate $(3,4)$. Although numerous

Correspondence to: Dr Qing Zhao, Department of Biochemistry, Guangzhou Medical University, 195 Dongfengxi Road, Guangzhou, Guangdong 510182, P.R. China

E-mail: zhaoqing66@yahoo.com

Key words: WIN55, 212-2, hepatocellular carcinoma, migration, cell cycle, cannabinoid treatment options exist, including surgical resection and chemotherapy, the prognosis of HCC remains poor (5). Identifying novel molecular targets, developing novel drugs and researching mechanism-based agents are required for the improvement of HCC treatment.

Cannabis sativa has been used medically for several centuries. Cannabinoids are the major effective compound present in Cannabis sativa. Numerous previous studies have demonstrated that cannabinoids exert cell growth inhibition and antitumor effects (6-11). Furthermore, the cannabinoid receptors, which consist of seven transmembrane spanning domains, have been cloned. Two cannabinoid receptors have been identified to date: Cannabinoid receptor 1 (CB1) and 2 (CB2). A previous study demonstrated that the cannabinoid, WIN55, 212-2 (WIN), inhibited the proliferation of LNCap prostate cancer cells via cell cycle arrest at the G0/G1 phase, and elucidated the underlying mechanism (11). Furthermore, WIN has been demonstrated to inhibit the cell cycle of the BEL7402 HCC cell line; however, its underlying mechanism remains to be elucidated (12). In addition, cannabinoids have been reported to inhibit the metastasis of non-small cell lung cancer (13). However, little is currently known regarding the role of synthetic cannabinoids in BEL7402 cell cycle and metastasis.

The present study demonstrated that treatment of BEL7402 HCC carcinoma cells with the cannabinoid receptor agonist, WIN, led to cell cycle arrest at the G0/G1 phase. Cell cycle arrest was associated with inactivation of extracellular signal-regulated kinases (ERK)1/2, increased expression of p27, and decreased expression of cyclin D1 and cyclin-dependent kinase (Cdk)4. Inhibiting CB2 with the CB2 antagonist, AM630, led to the inactivation of ERK1/2. Inhibition of ERK1/2 signaling by its inhibitor PD98059 also resulted in similar effects. The present study also aimed to determine the role of WIN on BEL7402 cell migration, and to explore the potential underlying mechanisms.

\section{Materials and methods}

Materials. R-(+)-[2,3-Dihydro-5-methyl-3[(4-morpholinyl) methyl]pyrrolo[1,2,3-de]-1,4-benzoxazinyl]-(1-naphthalenyl) methanone mesylate salt (WIN) and dimethyl sulfoxide 
(DMSO) were purchased from Sigma-Aldrich (St. Louis, MO, USA). The CB2 antagonist, AM630, was purchased from Tocris Bioscience (Bristol, UK). The CB2 selective agonist, JWH-015, was purchased from Enzo Life Sciences, Inc. (Farmingdale, NY, USA). The mitogen-activated protein kinase (MAPK) antagonist, PD98059, was purchased from Beyotime Institute of Biotechnology (Haimen, China). Rat polyclonal anti-CB2 antibodies were purchased from Abcam (Cambridge, MA, USA; cat no. ab3561; 1:200 dilution). Rabbit polyclonal anti-matrix metalloproteinase (MMP)9 antibodies were purchased from Rockland Immunochemicals Inc. (Philadelphia, PA, USA; cat no. 600-401-CU9; 1:1,000 dilution). Rabbit polyclonal anti-cyclin D1 (cat no. SC753; 1:300 dilution) and mouse monoclonal CDK4 (cat no. SC23896; 1:1,000 dilution) antibodies were purchased from Santa Cruz Biotechnology, Inc. (Dallas, TX, USA). Rabbit monoclonal phosphorylated (p)-p42/44 MAPK (ERK1/2) (Thr202/Tyr204) (cat no. 4094; 1:1,000 dilution) and rabbit monoclonal p-retinoblastoma (Rb) (cat no. 8516; 1:1,000 dilution) antibodies were purchased from Cell Signaling Technology, Inc. (Danvers, MA, USA). Rabbit polyclonal p27 (cat no. 25614-1-AP; 1:200 dilution), rabbit polyclonal E2F1 (cat no. 12334-1-AP; 1:300 dilution) and rabbit polyclonal $\beta$-actin (cat no. 20536-1-AP; 1:1,000 dilution) antibodies were purchased from Proteintech Group, Inc. (Chicago, IL, USA).

Cell culture. BEL7402 cells (Institute of Biochemistry and Cell Biology, Shanghai Institutes for Biological Sciences, Chinese Academy of Sciences, Shanghai, China) were cultured in RPMI-1640 medium (Gibco; Thermo Fisher Scientific, Inc., Waltham, MA, USA), supplemented with $10 \%$ (v/v) heat-inactivated fetal calf serum (Zhejiang Tianhang Biotechnology Co., Ltd., Hangzhou, China), $2 \mathrm{mM}$ L-glutamine, $100 \mathrm{U} / \mathrm{ml}$ penicillin and $100 \mu \mathrm{g} / \mathrm{ml}$ streptomycin (all from Beyotime Institute of Biotechnology), and incubated in a humidified atmosphere containing $5 \% \mathrm{CO}_{2}$.

Cell viability and anti-proliferation assay. BEL7402 cells were seeded into 96 -well plates at density of $5 \times 10^{3}$ cells/well in $100 \mu \mathrm{l}$ cell medium. The cells were allowed to adhere for $24 \mathrm{~h}$, and were subsequently treated with PD98059 at 0, 5, 10, 20,30 or $40 \mu \mathrm{M}$, or WIN at $0,5,10$ or $20 \mu \mathrm{M}$ for $24 \mathrm{~h}$. Subsequently, $20 \mu$ l Cell Counting kit- 8 solution (Nanjing KeyGen Biotech Co., Ltd., Nanjing, China) was added to each well and the culture was incubated for $1 \mathrm{~h}$ at $37^{\circ} \mathrm{C}$. All experiments were performed at least three times. The optical density values were read at $450 \mathrm{~nm}$ using a microplate reader (no. 680; Bio Rad Laboratories, Inc., Hercules, CA, USA).

Cell treatment. WIN55, 212-2, dissolved in DMSO, was used to treat the cells. For experiments, the cells were seeded at $60-70 \%$ confluence, allowed to adhere overnight and subsequently treated with the compounds. The final concentration of DMSO used was $0.1 \%(\mathrm{v} / \mathrm{v})$ for each treatment. For dose-dependent studies, BEL7402 cells were treated with WIN at 0,5 or $10 \mu \mathrm{M}$ final concentration for $24 \mathrm{~h}$ in serum-free medium. For the subsequent experiments, control cells were treated with vehicle alone, and the WIN groups were treated with $10 \mu \mathrm{M}$ WIN for $24 \mathrm{~h}$. To explore the role of the CB2 receptor in WIN-induced ERK1/2 inactivation, the cells were pretreated with $10 \mu \mathrm{M}$ AM630 for $0.5 \mathrm{~h}$ followed by incubation with both $10 \mu \mathrm{M}$ AM630 and $10 \mu \mathrm{M}$ WIN for $24 \mathrm{~h}$. To study CB2 selective agonist JWH-015-induced ERK1/2 inactivation, the cells were treated with $4 \mu \mathrm{M} \mathrm{JWH}-015$ for $24 \mathrm{~h}$, and the WIN group was treated with $10 \mu \mathrm{M}$ for $24 \mathrm{~h}$. To assess the role of ERK1/2 in cannabinoid receptor-induced cell growth inhibition, the cells were treated with $30 \mu \mathrm{M}$ ERK1/2 inhibitor, PD98059, for $24 \mathrm{~h}$.

Flow cytometric cell cycle analysis. The cells were grown to a density of $1 \times 10^{6}$ cells in $100 \mathrm{~mm}$ culture dishes and were treated for $24 \mathrm{~h}$ as described above. The cells were harvested by trypsinization, re-suspended in phosphate-buffered saline (PBS) and fixed in $70 \%$ (v/v) ethyl alcohol overnight at $4^{\circ} \mathrm{C}$. Cell cycle analysis was performed using a Cell Cycle kit (BestBio, Shanghai, China), and cell cycle distribution was evaluated using the FACSCalibur flow cytometer (BD Biosciences, Franklin Lakes, NJ, USA). The results were analyzed using ModFit LT software (version 3.2; Verity Software House, Topsham, ME, USA).

Cell migration assay. Cell migration was detected using Transwell migration and wound healing assays. For the Transwell assay, BEL7402 cells were treated with WIN at a concentration of 0,5 or $10 \mu \mathrm{M}$ for $24 \mathrm{~h}$. The cells were subsequently trypsinized and suspended in medium containing $2 \%$ fetal bovine serum (FBS). Cell suspensions $(200 \mu \mathrm{l})$, containing $2 \times 10^{5}$ cells, were seeded into the upper chamber of a 24-well Transwell (pore size, $8 \mu \mathrm{m}$; Corning Incorporated, Corning, NY, USA). The Transwells were then inserted into a 24-well plate, containing $600 \mu 1$ RPMI-1640 medium, supplemented with $10 \% \mathrm{FBS}$ and incubated at $37^{\circ} \mathrm{C}$ in a humidified atmosphere for $24 \mathrm{~h}$, in order to allow the BEL7402 cells to migrate. Cells on the upper side of the filter (not migrated) were removed with cotton swabs. Migrated cells on the lower side of the filter were fixed and stained with DAPI (Beyotime Institute of Biotechnology). The number of BEL7402 cells that had migrated to the lower surface of the membrane was counted in five random and non-repeated high-power fields under a fluorescence microscope (Nikon Eclipse Ti-S; Nikon, Tokyo, Japan). The average number of migrated cells for each group was subsequently calculated. Each assay was performed in triplicate wells. Cell migration was also assessed using a scratch wound healing assay. To visualize the migration of BEL7402 cells into artificial wounds, the BEL7402 cells were seeded into 6-well plates and allowed to grow for $24 \mathrm{~h}$ in RPMI-1640 medium, supplemented with 10\% FBS. A $200 \mu \mathrm{l}$ plastic pipette tip was used to gently scratch the cell monolayer, in order to create a cell-free area. Subsequently, the cells were washed extensively with PBS to remove cellular debris. The cells were then incubated with 0,5 or $10 \mu \mathrm{M}$ WIN in serum-free RPMI-1640 for $24 \mathrm{~h}$. Wound closure was monitored after staining with DAPI. Images of marked regions along the wounded area were obtained using an inverted microscope (Nikon Eclipse Ti-S) attached to a camera.

Western blot analysis. Once the cells were treated with the indicated compounds, the total protein was extracted from the cells by washing in PBS and incubating for $20 \mathrm{~min}$ in ice-cold lysis buffer, supplemented with a protease and phosphatase 
A
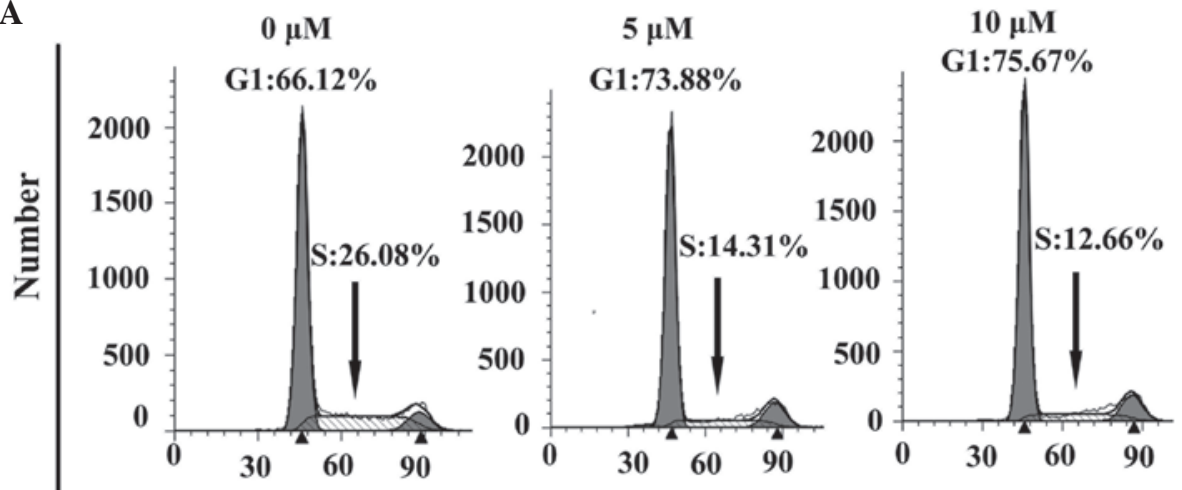

Channels (Propidium Iodide-APropidium Iodide-A)

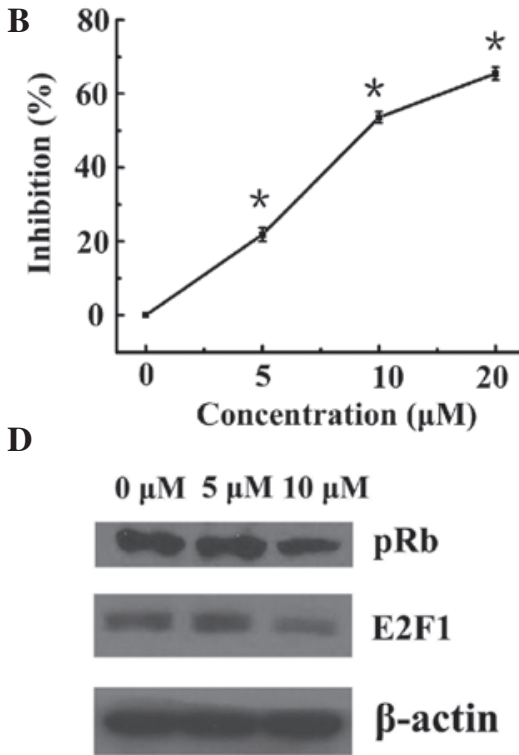

C

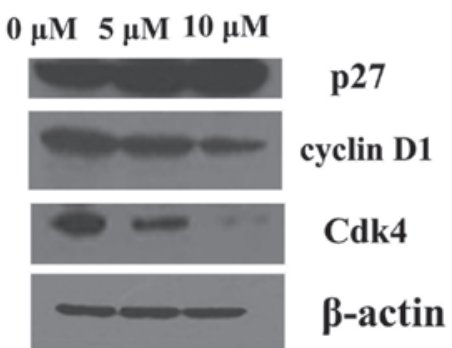

Е $\quad 0 \mu \mathrm{M} 5 \mu \mathrm{M} 10 \mu \mathrm{M}$

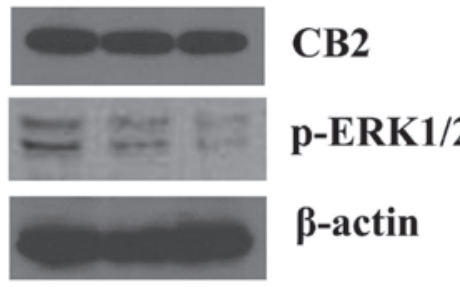

Figure 1. Effects of WIN treatment on cell cycle distribution, cell proliferation and protein expression in BEL7402 hepatocellular carcinoma cells. (A) The effects of WIN treatment on cell cycle distribution were determined by flow cytometry in BEL7402 cells. The labeled cells were analyzed using a FACSCalibur flow cytometer, and the percentage of cells in the G0/G1 and S phases was calculated using ModFit LT software. The data presented are representative of a typical experiment repeated three times. (B) BEL7402 cell proliferation was inhibited following treatment with various concentrations of WIN for $24 \mathrm{~h}$. Values are expressed as the mean \pm standard deviation. ${ }^{*} \mathrm{P}<0.05$ vs. untreated cells. The effects of WIN treatment on the protein expression levels of $(\mathrm{C})$ p 27 , cyclin $\mathrm{D} 1$ and cyclin-dependent kinase (Cdk4); (D) retinoblastoma $(\mathrm{Rb})$ and E2F1; and (E) cannabinoid receptor (CB)2 and phosphorylated extracellular signal-regulated kinases (p-ERK)1/2 in BEL7402 cells. The cells were treated with dimethyl sulfoxide alone or with the specified concentrations of WIN and total cell lysates were prepared for immunoblot analysis. The data presented are representative a typical experiment repeated three times. WIN, WIN55, 212-2.

inhibitor cocktail (Roche Diagnostics Ltd., Shanghai, China). The cells were sonicated three times for $10 \mathrm{sec}$ and the protein concentrations were subsequently determined using a bicinchoninic acid assay (Beyotime Institute of Biotechnology). Equal quantities of protein samples ( $80 \mu \mathrm{g} / \mathrm{lane})$ were separated by $12 \%$ SDS-PAGE (SDS-PAGE kit; Beijing Dingguochangsheng Biotechnology Co. Ltd., Beijing, China) and then electrotransferred onto polyvinylidene fluoride membranes (EMD Millipore, Bedford, MA, USA). Non-specific sites on the blots were blocked by a $1 \mathrm{~h}$ incubation at room temperature with blocking buffer (5\% nonfat dry milk, 1\% Tween 20 in $20 \mathrm{mmol} / \mathrm{l}$ Tris-buffered saline; $\mathrm{pH}$ 7.6). The membranes were then incubated with the specific primary antibodies overnight at $4^{\circ} \mathrm{C}$, followed by a $1 \mathrm{~h}$ incubation at room temperature with horseradish peroxidase-conjugated secondary antibodies (Proteintech Group, Inc.). The blots were developed using enhanced chemiluminescence reagents (Beyotime Institute of Biotechnology), according to the manufacturer's protocol.
Statistical analysis. The results are expressed as the mean \pm standard deviation. The data were analyzed by Student's t-test in order to determine statistical significance. Statistical analyses were performed using SPSS 13.0 (SPSS, Inc., Chicago, IL, USA). $\mathrm{P}<0.05$ was considered to indicate a statistically significant difference.

\section{Results}

WIN results in cell cycle arrest at G0/G1 phase and inhibition of proliferation. The present study aimed to test the hypothesis that WIN inhibited the proliferation of BEL7402 cells via cell cycle arrest. A DNA cell cycle analysis was performed, in order to assess the effects of WIN treatment on cell cycle distribution. As shown in Fig. 1A, treatment with WIN resulted in a dose-dependent accumulation of cells in G1 phase of the cell cycle $(66.12,73.88$ and $75.67 \%$ of cells in G1 phase following treatment with 0,5 and $10 \mu \mathrm{M}$ WIN, respectively), as 
A
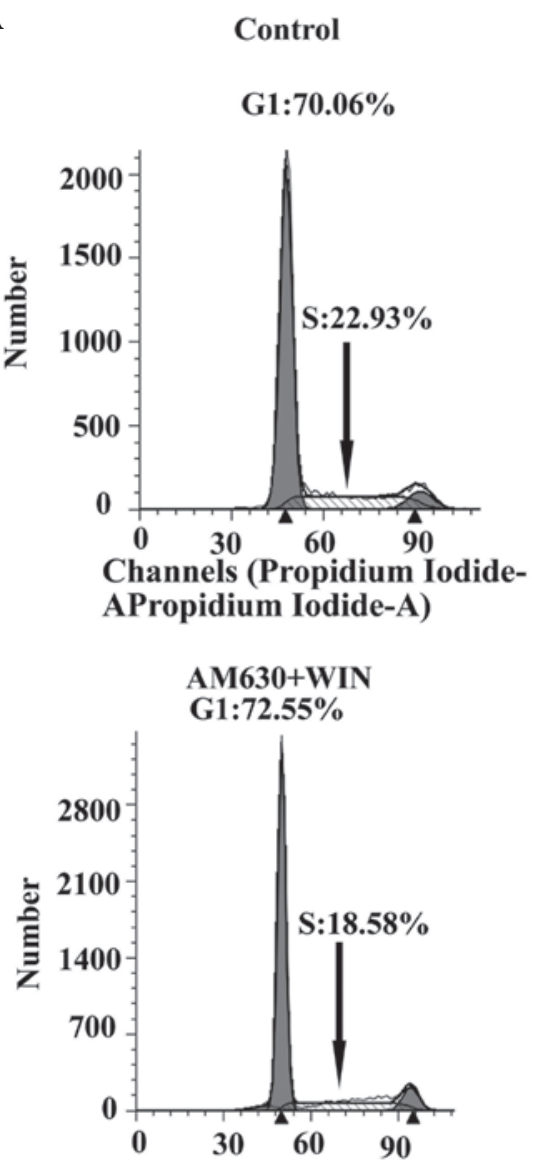

Channels (Propidium IodideAPropidium Iodide-A)

B

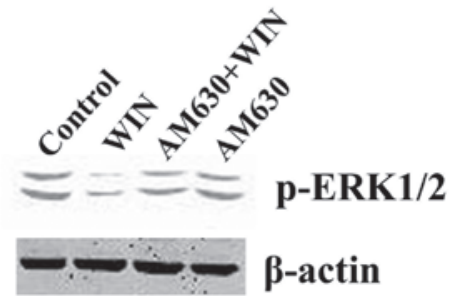

WIN

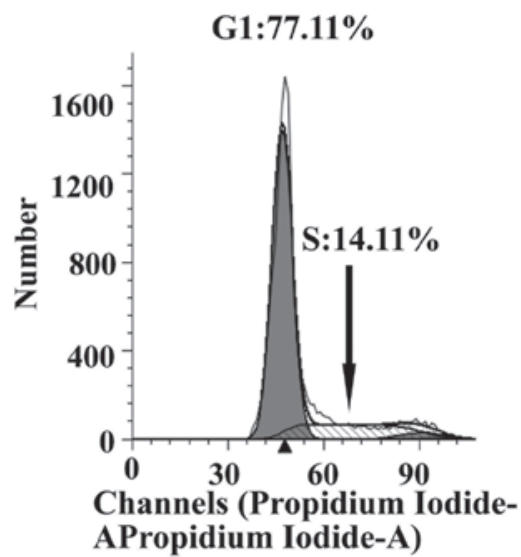

AM630

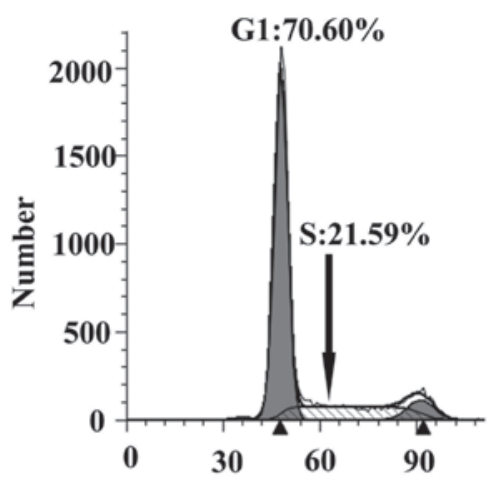

Channels (Propidium IodideAPropidium Iodide-A)

C

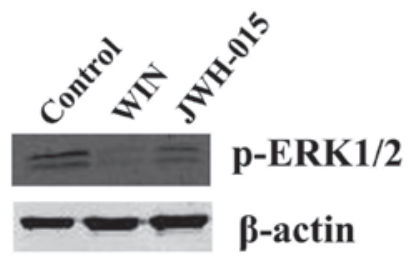

Figure 2. Effects of CB2 on the WIN-induced cell cycle arrest of BEL7402 hepatocellular carcinoma cells. (A) Effects of the CB2 antagonist, AM630, on the (A) cell cycle distribution and (B) protein expression levels of p-ERK1/2 in BEL7402 cells. (C) Effects of CB2 selective agonist, JWH-015, on the protein expression levels of p-ERK1/2 in BEL7402 cells. CB, cannabinoide receptor; WIN, WIN55, 212-21 p-ERK, phosphorylated-extracellular signal-regulated kinases.

compared with vehicle treatment. In addition, treatment with WIN led to a dose-dependent decrease in the number of cells in $\mathrm{S}$ phase of the cell cycle $(26.08,14.31$ and $12.66 \%$ cells in $\mathrm{S}$ phase following treatment with 0,5 and $10 \mu \mathrm{M}$ WIN, respectively). As shown in Fig. 1B, WIN inhibited the proliferation of BEL7402 cells in a dose-dependent manner.

WIN-induced cell cycle arrest is mediated via the upregulation of 27 and concomitant downregulation of cyclin D1 and $C d k 4$. Since the present study demonstrated that treatment of BEL7402 cells with WIN resulted in a G1 phase arrest, the effects of WIN on the cell cycle regulatory molecules that operate in G1 phase of the cell cycle were assessed. The present study also investigated the role of the Cdk inhibitor (Cki) cyclin Cdk machinery in the WIN-mediated G1 phase cell cycle arrest of BEL7402 cells. A marked increase in the protein expression of p27 was detected following treatment with WIN at 5 and $10 \mu \mathrm{M}$ doses (Fig. 1C). Using western blot analysis, the effects of WIN on the protein expression levels of cyclin D1 and Cdk4, which are known to be regulated by p27, were investigated. Treatment of BEL7402 cells with WIN led to a dose-dependent decrease in the protein expression levels of cyclin D1 and Cdk4 (Fig. 1C).

WIN downregulates the levels of $p-R b$ and E2F1. The present study also detected the effects of WIN on the protein expression levels of $\mathrm{pRb}$ and E2F1. Western blot analysis revealed that treatment of BEL7402 cells with WIN led to a significant decrease in the protein expression of $\mathrm{pRb}$ (Fig. 1D). Since $\mathrm{pRb}$ controls the cell cycle via binding to and inhibiting the E2F1 
A

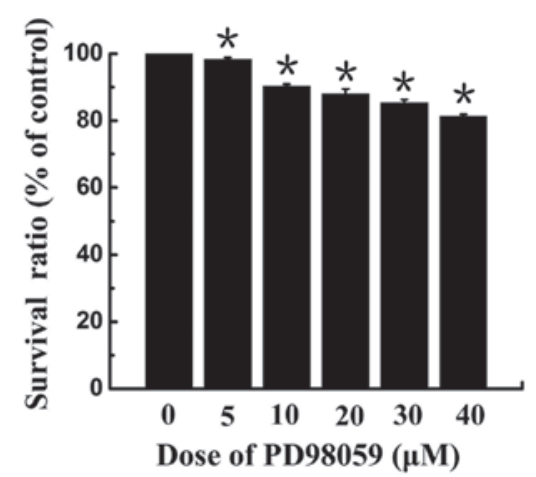

B

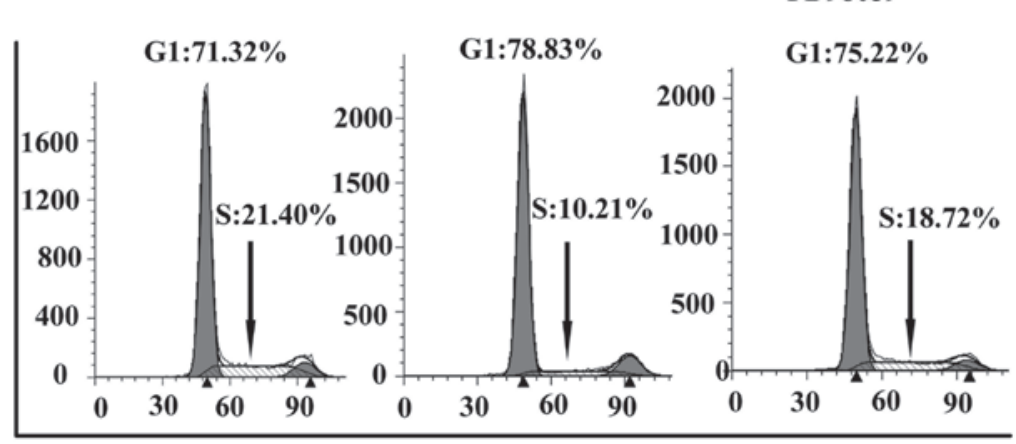

Channels (Propidium Iodide-APropidium Iodide-A)

C

Control WIN PD98059
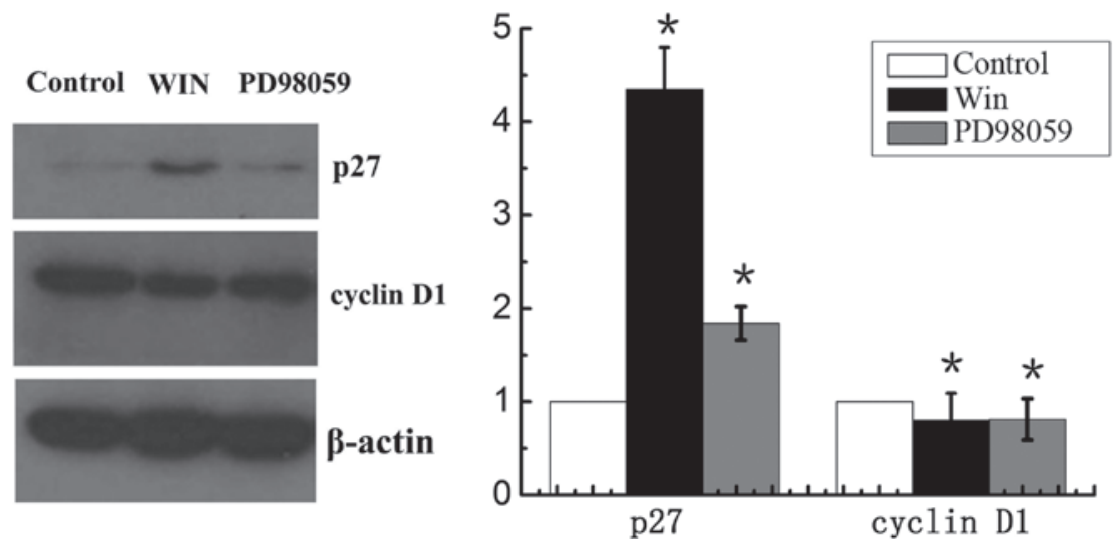

Figure 3. Effects of WIN and ERK1/2 inhibitor on cell cycle distribution, and cyclin D1 and p27 expression in BEL7402 hepatocellular carcinoma cells. (A) Effects of ERK1/2 inhibitor, PD98059, on the cell viability of BEL2407 cells. (B) Effects of the ERK1/2 inhibitor, PD98059, on the cell cycle distribution of BEL7402 cells. Cell cycle analysis was performed by flow cytometry and the labeled cells were analyzed using a FACScan flow cytometer, and the percentage of cells in the G0/G1, S and G2/M phases was calculated using ModFit LT software. The data are representative of a typical experiment repeated three times. (C) Effects of ERK1/2 inhibitor PD98059 on the protein expression levels of p27 and cyclin D1 in BEL7402 cells. Total cell lysates were prepared for immunoblot analysis. The blot shown is representative of a typical experiment repeated three times. Expression levels were quantified by densitometric analysis with normalization to $\beta$-actin. Values are expressed as the mean \pm standard deviation. ${ }^{*} \mathrm{P}<0.05$ vs. untreated cells. WIN, WIN55, 212-2; ERK, extracellular signal-regulated kinase.

transcription factor, the protein expression of E2F1 was also detected. As shown in Fig. 1D, treatment of BEL7402 cells with WIN led to a dose-dependent decrease in E2F1 expression.

WIN-induced inactivation of ERK results in cell cycle arrest via $C B 2$. A significant inactivation of ERK1/2 was detected when the BEL7402 cells were treated with WIN at a dose of 5 and $10 \mu \mathrm{M}$ (Fig. 1E). To confirm that ERK1/2 inactivation was cannabinoid receptor-mediated, the BEL7401 cells were pretreated with the CB2 antagonist, AM630, followed by treatment with both AM630 and WIN. As shown in Fig. 2B, no alteration in the activation of ERK1/2 was observed when the cells were treated with the antagonist alone, as compared with the control group. However, treatment with WIN led to a marked inactivation of ERK1/2. When the antagonist was co-administered with WIN, an increase in the protein expression of p-ERK1/2 was detected, as compared with in the cells treated with WIN alone. As shown in Fig. 2A, when the cells were treated with the antagonist alone, no change in the number of cells in G1 phase (70.60\%) and $\mathrm{S}$ phase $(21.59 \%)$ was observed, as compared with the control group (G1 phase, $70.06 \%$; S phase, 22.93\%). Treatment with WIN led to a significant inhibition in cell cycle progression (G1 phase, 77.11\%; S phase, 14.11\%). However, when the antagonist was co-administered with WIN, the inhibition in cell cycle progression was attenuated (G1 phase, $72.55 \%$; $\mathrm{S}$ phase, 18.58\%). Furthermore, in the BEL7402 cells treated with the CB2 selective agonist, JWH-015, the protein expression of p-ERK1/2 was significantly decreased (Fig. 2C).

WIN-inducedinactivation of ERK $1 / 2$ results in cell growth inhibition and cell cycle arrest. To confirm the role of p-ERK1/2 in cannabinoid receptor-induced cell growth inhibition, the BEL7402 cells were treated with $30 \mu \mathrm{M}$ ERK1/2 inhibitor, PD98059, for $24 \mathrm{~h}$. Treatment with PD98059 alone resulted in a decrease in the viability of BEL7402 cells in a dose-dependent manner; however, $85.4 \pm 0.9 \%$ cells survived following treatment with $40 \mu \mathrm{M}$ PD 98059 (Fig. 3A). The present study demonstrated that treatment of BEL7402 cells with WIN resulted in G1 phase cell cycle arrest. To determine whether the cell cycle arrest was mediated by inactivation of ERK1/2, a DNA cell cycle analysis was performed. As shown in Fig. 3B, inhibiting the activation of ERK1/2 using the inhibitor PD98059 resulted in an increase in the number of cells in G1 phase of the cell cycle (75.22\%), as compared with the control group (71.32\%). In addition, the number of cells in S phase of cell cycle was decreased (18.72\%), as compared with the control group $(21.40 \%)$. Treatment with WIN led to an accumulation of cells in G1 phase $(78.83 \%)$ and a reduction 
A

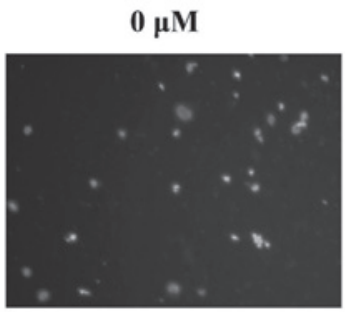

$5 \mu \mathrm{M}$

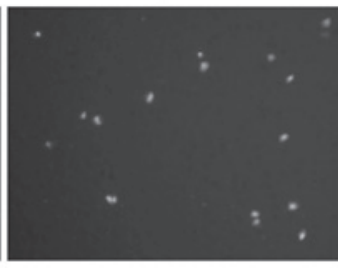

B

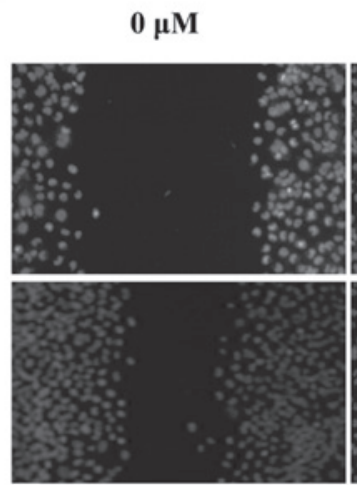

C

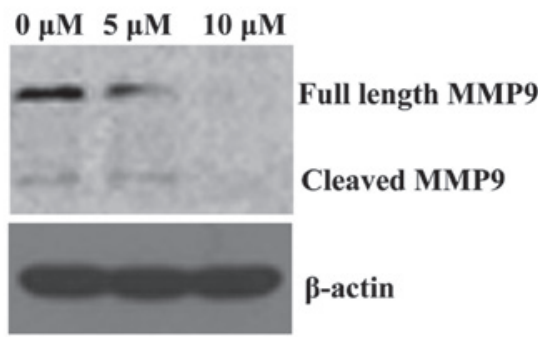

$5 \mu \mathrm{M}$

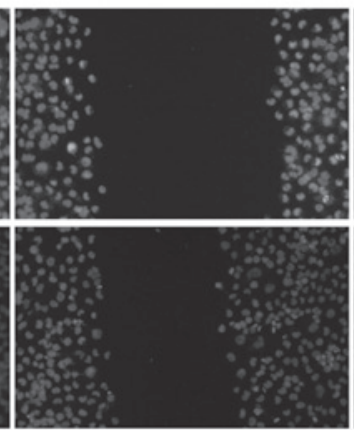

D

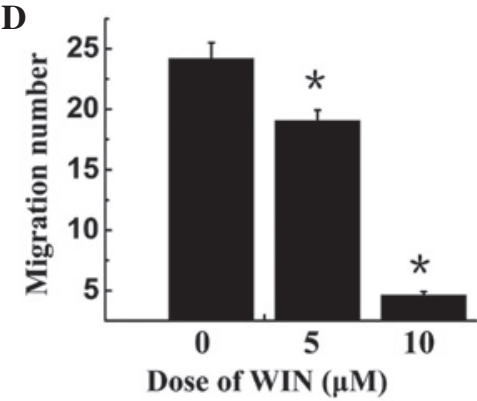

Figure 4. Effects of WIN on the migration of BEL7402 hepatocellular carcinoma cells. (A) Representative migration images of BEL7402 cells in a Transwell migration system $24 \mathrm{~h}$ following serum induction (original magnification, x400). (B) Representative migration images of BEL7402 cells in a wound healing assay after $24 \mathrm{~h}$. The cells were treated with various doses of WIN or dimetyl sulfoxide prior to the assay (original magnification, $\mathrm{x} 400$ ). (C) Effects of WIN treatment on the protein expression levels of MMP-9 in BEL7402 cells. Total cell lysates were prepared for immunoblot analysis. The data are representative of a typical experiment repeated three times. (D) Quantification of the effects of WIN on the migration of BEL7402 cells ("P $<0.05$, vs. $0 \mu \mathrm{M}$ WIN; $\mathrm{n}=15$ ). Values were determined from five random and non-repeated high-power fluorescence microscopy fields with assays performed in triplicate and are expressed as the mean \pm standard deviation. WIN, WIN55, 212-2; MMP, matrix metalloproteinase.

of cells in S phase (10.21\%). In addition, the present study determined the effects of ERK1/2 on the expression of $\mathrm{p} 27$, a cell cycle regulatory molecule that operates in G1 phase of the cell cycle, and cyclin D1, which is associated with cell proliferation. Treatment with WIN increased the expression of p27, and treatment with PD98059 also increased the expression of p27 (Fig. 3C). Furthermore, treatment with WIN or PD98059 markedly inhibited the expression of cyclin D1 in the BEL7402 cells (Fig. 3C).

WIN inhibits BEL7402 cell migration via MMP-9 downregulation. The present study performed Transwell and wound healing assays using BEL7402 cells. As shown in Fig. 4A and D, cell migration was significantly decreased following treatment with WIN in a dose-dependent manner; the number of migrated cells was $24.20 \pm 1.31,19.07 \pm 0.88$, and $4.60 \pm 0.29$ following treatment with 0,5 and $10 \mu \mathrm{M}$ WIN, respectively. The wound healing assay demonstrated that BEL7402 cells treated with WIN invaded the wound more slowly compared with the control group, in a dose-dependent manner (Fig. 4B). MMPs may be associated with the impaired migration of WIN-treated cells. To explore this hypothesis, the present study examined the protein expression of MMP-9. As shown in Fig. 4C, MMP-9 was significantly reduced in the cells treated with WIN, as compared with the control group.

\section{Discussion}

Cannabinoids and their derivatives have recently attracted attention in the treatment of cancer due to their diverse abilities, including anti-inflammation, cell growth inhibition and antitumor properties $(6,10)$. Previous studies have suggested that cannabinoid receptors may be an essential target for the treatment of cancer $(14,15)$. Our previous study indicated that WIN-induced apoptosis of BEL7402 HCC cells may be mediated via the $\mathrm{CB} 2$ receptor; therefore, this receptor may be considered as a potential target for the treatment of HCC (12). A previous study suggested that cannabinoids inhibit 


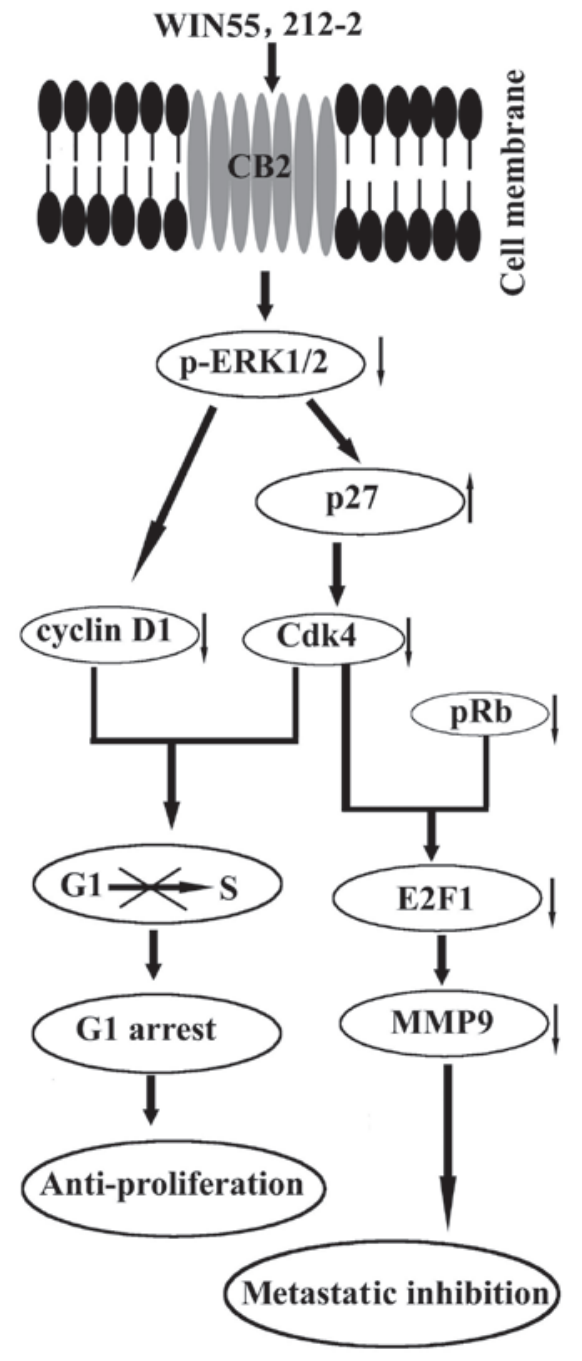

Figure 5. Schematic diagram of WIN55, 212-2-mediated cell cycle dysregulation and metastatic inhibition. $\mathrm{CB}$, cannabinoid receptor; p-ERK, phosphorylated-extracellular signal-regulated kinases; Cdk, cyclin-dependent kinase; $\mathrm{pRb}$, retinoblastoma protein; MMP, matrix metalloproteinase.

non-small cell lung cancer growth and metastasis; however, little is currently known regarding the effects and underlying mechanisms of cannabinoids on non-small cell lung cancer proliferation and migration (13). The present study aimed to determine the mechanism underlying the antiproliferative and antimigratory effects of the cannabinoid agonist, WIN, against HCC. The results of the present study demonstrated for the first time, to the best of our knowledge, that the treatment of BEL7402 cells with WIN was able to inactivate ERK1/2, resulting in cell cycle dysregulation and G1 arrest. In addition, treatment of BEL7402 cells with WIN decreased the expression levels of MMP-9, leading to the inhibition of migration.

ERK $1 / 2$ has a dual function and is involved in cell cycle arrest and proliferation. Inactivation of ERK1/2, and cell death and proliferation depend on numerous factors. A previous study demonstrated that cell cycle progression was mediated by the ERK1/2 and p27 pathway in prostate cancer (11). In addition, treatment with the ERK1/2 inhibitor, PD98059, downregulated the protein expression levels of p27 and cyclin D1 in BEL7402 cells, and led to G1 phase cell cycle arrest. These data suggested that the ERK1/2 pathway may be involved in the WIN-mediated cell cycle arrest in BEL7402 cells. Although certain cannabinoids are able to function via transiently activated vanilloid receptors or lipid rafts, the majority of cannabinoids act predominantly via cannabinoid receptors (16). The expression levels of CB2 have previously been shown to be higher in BEL7402 cells, as compared with in LO2 normal human hepatocytes (12). The present study demonstrated that WIN significantly downregulated the expression levels of ERK1/2 in BEL7402 cells; however, treatment with the CB2 antagonist AM630, was able to attenuate the inhibition. Treatment with WIN resulted in a marked accumulation of BEL7402 cells in G1 phase; however, treatment with the CB2 antagonist AM630, attenuated this effect. Furthermore, the CB2 selective agonist, JWH-015, decreased the protein expression levels of ERK1/2 in BEL7402 cells. These data suggested that cell cycle dysregulation in BEL7402 cells following treatment with WIN was regulated via CB2, and the p-ERK1/2 and p27 pathway.

It is well established that uncontrolled cellular growth and metastatic reoccurrence are responsible for the development of the majority of cancer types, including HCC. Therefore, agents that can modulate cell cycle and metastasis may be useful in the management and treatment of cancer. Consistent with this notion, developing novel targets and mechanism-based anti-proliferative and anti-migratory agents for the management of HCC is essential. One of the most promising areas of recent cannabinoid research is the ability to control cell growth (8). Numerous studies have demonstrated that the cannabinoid-mediated inhibition of growth may be cell cycle-dependent $(11,17)$. Therefore, the present study analyzed the effects of WIN on the proportion of cells in various phases of the cell cycle. WIN was able to induce a dose-dependent accumulation of cells in G1 phase of the cell cycle, and also resulted in an inhibition of cell proliferation. Inhibition of the cell cycle has previously been suggested as a target for the management of cancer (11). The present study also investigated the role of the $\mathrm{Cki}$-cyclin-Cdk machinery in the WIN-mediated G1 phase cell cycle arrest of BEL7402 cells. The eukaryotic cell cycle is regulated by protein kinase complexes, which are comprised of cyclins (the regulatory subunit), which bind to Cdks (catalytic subunit), in order to form active cyclin-Cdk complexes. Cdk activity is additionally regulated by small proteins, known as $\mathrm{Ckis}$, which include p27. It has been reported that $\mathrm{Ckis}$ inhibit the kinase activities associated with cyclin-Cdk complexes; therefore, modulating the phosphorylation events that have a critical role in the progression of the cell cycle (18). A previous study demonstrated that cell cycle progression through the G0/G1 phase is regulated by p27 (19). The present study revealed that treatment of BEL7402 cells with WIN led to an increase in the expression levels of p27 and a decrease in the expression levels of cyclin D1 and Cdk4. These results indicated that cell cycle dysregulation in BEL7402 cells following treatment with WIN may be regulated via the p27, cyclin D1 and Cdk4 pathway.

It has previously been reported that downregulation of $\mathrm{Cdk} 4$ results in phosphorylation and inactivation of $\mathrm{pRb}$, which can in turn downregulate members of the E2F family and inhibit the transcription of genes required for $\mathrm{S}$ phase progression (20). 
Progression of S phase in the cell cycle is accomplished by transcriptional activation of E2F target genes via phosphorylation of pocket proteins by $\mathrm{Cdks}$ (18). E2F1 is able to regulate the expression of MMP-9, and therefore induce migration (21-23). MMPs are involved in cell migration and are frequently upregulated in cancer cells (24). The present study demonstrated that treatment of BEL7402 cells with WIN downregulated the expression levels of $\mathrm{pRb}$ and E2F1. Protein expression levels of MMP-9 were downregulated in BEL7402 cells following treatment with WIN, which significantly impaired their migratory capability. Furthermore, Transwell and wound healing assays indicated that treatment of BEL7402 cells with WIN significantly inhibited the metastatic capability of the cells. These results indicated that metastatic inhibition of BEL7402 cells by WIN may be regulated via the $\mathrm{Cdk} 4$, pRb, E2F1 and MMP-9 pathway.

In conclusion, the results of the present study suggested that WIN may inhibit metastasis via the MMP-9 pathway and induce cell cycle arrest via ERK1/2 inactivation in BEL7402 HCC cells. These results provided a basis for the application of WIN in the treatment of HCC.

\section{Acknowledgements}

This work was supported by the National Science Foundation of China (no. 81372466), International Conference on Transcriptomics, the 2014 undergraduate and graduate training innovation project of Guangdong province (GDJG20141136,2014JGXM-MS20) and the project of Guangzhou Municipality Bureau of Education (no. 11A033).

\section{References}

1. Lin H, van den Esschert J, Liu C and van Gulik TM: Systematic review of hepatocellular adenoma in China and other regions. J Gastroenterol Hepatol 26: 28-35, 2011.

2. Tanaka M, Katayama F, Kato H, Tanaka H, Wang J, Qiao YL and Inoue $\mathrm{M}$ : Hepatitis $\mathrm{B}$ and $\mathrm{C}$ virus infection and hepatocellular carcinoma in China: A review of epidemiology and control measures. J Epidemiol 21: 401-416, 2011.

3. Sia D and Villanueva A: Signaling pathways in hepatocellular carcinoma. Oncology 81 (Suppl 1): 18-23, 2011.

4. Qin LX and Tang ZY: Recent progress in predictive biomarkers for metastatic reccurrence of human hepatocellular carcinoma: A review of the literature. J Cancer Res Clin Oncol 130: 497-513, 2004.

5. Tabrizian P, Franssen B, Jibara G, Sweeney R, Sarpel U, Schwartz M and Labow D: Cytoreductive surgery with or without hyperthermic intraperitoneal chemotherapy in patients with peritoneal hepatocellular carcinoma. J Surg Oncol 110 786-790, 2014

6. Hermanson DJ and Marnett LJ: Cannabinoids, endocannabinoids, and cancer. Cancer Metastasis Rev 30: 599-612, 2011.

7. Bowles DW, O'Bryant CL, Camidge DR and Jimeno A: The intersection between cannabis and cancer in the United States. Crit Rev Oncol Hematol 83: 1-10, 2012.
8. Giuliano M, Pellerito O, Portanova P, Calvaruso G, Santulli A, De Blasio A, Vento R and Tesoriere G: Apoptosis induced in HepG2 cells by the synthentic cannabinoid WIN: Involvement of the transcription factor PPARgamma. Biochimie 91: 457-465, 2009.

9. Calvaruso G, Pellerito O, Notaro A and Giuliano $\mathrm{M}$ : Cannabinoid-associated cell death mechanisms in tumor models (review). Int J Oncol 41: 407-413, 2012.

10. Chakravarti B, Ravi J and Ganju RK: Cannabinoids as therapeutic agents in cancer: Current status and future implications. Oncotarget 5: 5852-5872, 2014.

11. Sarfaraz S, Afaq F, Adhami VM, Malik A and Mukhtar H: Cannabinoid receptor agonist-induced apoptosis of human prostate cancer cells LNCaP proceeds through sustained activation of ERK1/2 leading to G1 cell cycle arrest. J Biol Chem 281: 39480-39491, 2006.

12. Hong Y, Zhou Y, Wang Y, Xiao S, Liao DJ and Zhao Q: PPAR mediates the effects of WIN55,212-2, an synthetic cannabinoid, on the proliferation and apoptosis of the BEL-7402 hepatocarcinoma cells. Mol Biol Rep 40: 6287-6293, 2013.

13. Preet A, Qamri Z, Nasser MW, Prasad A, Shilo K, Zou X, Groopman JE and Ganju RK: Cannabinoid receptors, CB1 and CB2, as novel targets for inhibition of non-small cell lung cancer growth and metastasis. Cancer Prev Res (Phila) 4: 65-75, 2011.

14. Xian XS, Park H, Choi MG and Park JM: Cannabinoid receptor agonist as an alternative drug in 5-fluorouracil-resistant gastric cancer cells. Anticancer Res 33: 2541-2547, 2013.

15. Brandi J, Dando I, Palmieri M, Donadelli M and Cecconi D: Comparative proteomic and phosphoproteomic profiling of pancreatic adenocarcinoma cells treated with $\mathrm{CB} 1$ or CB2 agonists. Electrophoresis 34: 1359-1368, 2013.

16. Dainese E, Oddi S, Bari M and Maccarrone M: Modulation of the endocannabinoid system by lipid rafts. Curr Med Chem 14: 2702-2715, 2007

17. Zeni O, Sannino A, Romeo S, Micciulla F, Bellucci S and Scarfi MR: Growth inhibition, cell-cycle alteration and apoptosis in stimulated human peripheral blood lymphocytes by multiwalled carbon nanotube buckypaper. Nanomedicine (Lond) 10: 351-360, 2015.

18. Sánchez I and Dynlacht BD: New insights into cyclins, CDKs, and cell cycle control. Semin Cell Dev Biol 16: 311-321, 2005.

19. Kumar P and Wood C: Kaposi's sarcoma-associated herpesvirus transactivator Rtainduces cell cycle arrest in G0/G1 phase by stabilizing and promoting nuclear localization of p27kip. J Virol 87: 13226-13238, 2013.

20. Deshpande A, Sicinski P and Hinds PW: Cyclins and cdks in development and cancer: A perspective. Oncogene 24: 2909-2915, 2005

21. Ma X, Gao Y, Fan Y, Ni D, Zhang Y, Chen W, Zhang P, Song E, Huang Q, Ai Q, et al: Overexpression of E2F1 promotes tumor malignancy and correlates with TNM stages in clear cell renal cell carcinoma. PLoS One 8: e73436, 2013.

22. Johnson JL, Pillai S, Pernazza D, Sebti SM, Lawrence NJ and Chellappan SP: Regulation of matrix metalloproteinase genes by E2F transcription factors: Rb-Raf-1 interaction as a novel target for metastatic disease. Cancer Res 72: 516-526, 2012.

23. Pillai S, Kovacs M and Chellappan S: Regulation of vascular endothelial growth factor receptors by $\mathrm{Rb}$ and $\mathrm{E} 2 \mathrm{~F} 1$ : Role of acetylation. Cancer Res 70: 4931-4940, 2010.

24. Lukaszewicz-Zając M, Mroczko B and Szmitkowski M: Gastric cancer - The role of matrix metalloproteinases in tumor progression. Clin Chim Acta 412: 1725-1730, 2011. 